\title{
Hyperbolic geometry of multiply twisted knots
}

\author{
Jessica S. Purcell
}

\begin{abstract}
We investigate the geometry of hyperbolic knots and links whose diagrams have a high amount of twisting of multiple strands. We find information on volume and certain isotopy classes of geodesics for the complements of these links, based only on a diagram. The results are obtained by finding geometric information on generalized augmentations of these links.
\end{abstract}

\section{Introduction}

By Mostow-Prasad rigidity and work of Gordon and Luecke [10], the hyperbolic structure on the complement of a hyperbolic knot is a knot invariant, and ought to be useful in problems of knot and link classification. In practice, this structure seems difficult to compute.

In recent years, some geometric properties of hyperbolic knots and links have been discovered for links admitting certain types of diagrams, such as alternating links [16] and highly twisted knots and links [9, 22, 23]. However, many knots that are of interest to topologists and hyperbolic geometers do not fall into these classes. These include Berge knots [3-5], twisted torus knots and Lorenz knots [6], which contain many of the smallest volume hyperbolic knots [7]. These knots often have diagrams that are highly non-alternating, with few twists per twist region, but contain regions where multiple strands of the diagram twist around each other some number of times. We would like to be able to understand and estimate geometric properties of these "multiply twisted" knots and links, given only a diagram, but currently we do not have the tools to do so.

In this paper, we take a first step toward such an understanding. We investigate the geometry of knots and links with diagrams with a high amount of twisting of multiple strands. We find information on the geometry of these knots, including volume bounds and certain isotopy classes of geodesics, based only on a diagram.

The results are obtained by augmenting the knot and link diagrams. That is, we encircle each twist of multiple strands by a simple closed curve, unknotted in $S^{3}$. The resulting link is called a generalized augmented link, 
generalizing a construction of Adams in which two twisting strands are encircled by an unknotted component [1]. When one performs $1 / n$ Dehn filling on the augmentation components of these links, one adds $n$ full twists to the strands. All diagrams can be obtained by such twisting. (See Section 2 for a more careful discussion.) Hence geometric information on a generalized augmented link, combined with geometric information under Dehn filling, leads to geometric results on knot complements.

Regular augmented links have a very nice hyperbolic structure, including a decomposition into right angled ideal polyhedra, first written down by Agol and Thurston [16, Appendix]. Generalized augmented links do not have as nice structure, but still contain enough symmetry to obtain results. To determine geometric information on Dehn fillings of these manifolds, one may turn to results on cone deformations due to Hodgson and Kerckhoff [11-13], or hyperbolike filling of Agol [2] and Lackenby [15], or volume change results due to Futer, Kalfagianni, and Purcell [9].

We have investigated generalized augmented links elsewhere. In [24], we bounded the lengths of certain slopes on these links, and showed that many knots obtained by their Dehn fillings have meridian length approaching 4 from below. With Futer and Kalfagianni, in [8] we investigated properties of volumes of a very particular class of these links. Here, we broaden the results to larger classes of knots and links.

In Section 2, we give careful definitions of the terms generalized twist region and generalized augmented link, and show that a generalized augmented link admits a reflection. We use this to give information about certain slope lengths on the link in Section 3. This implies, among other things, that many of these knots are hyperbolic, as follows.

Theorem 3.2. Let $K$ be a knot or link in $S^{3}$ which has a diagram $D$ containing at least six half-twists in each generalized twist region, such that the corresponding generalized augmented link is hyperbolic. Then $S^{3}-K$ is also hyperbolic.

Next, in Section 4, we examine the volumes of generalized augmented links, and show that there exist lower bounds on volumes of many knots based only on the number of generalized twist regions in a diagram. The main result is the following.

Theorem 4.1. Let $K$ be a knot or link in $S^{3}$ which has a diagram $D$ with at least seven half-twists in each generalized twist region, and such that the corresponding generalized augmented link is hyperbolic. Let $\mathrm{tw}(D)$ denote 
the number of generalized twist regions in the maximal twist region selection. Then

$$
\operatorname{vol}\left(S^{3}-K\right) \geq 0.64756(\operatorname{tw}(D)-1)
$$

Finally, in Section 5, we examine the question of geodesics in these link complements. Kuhlmann showed that any cusped hyperbolic manifold admits infinitely many simple closed geodesics [14], and identified isotopy classes of geodesics in the figure-eight knot complement [19]. However, as far as we are aware these are the only previously known explicit results identifying isotopy classes of closed geodesics in knot complements. It seems to be a difficult problem to identify isotopy classes of closed geodesics given only a diagram of a knot. Our main result of Section 5 addresses this problem. We show that certain closed curves in a diagram of a generalized augmented link are isotopic to geodesics under restrictions depending only on the link diagram. This is the content of Theorem 5.2.

Note that the focus of this paper is on geometric information for hyperbolic generalized augmented links and their Dehn fillings. In a companion paper, we discuss results for generalized augmented links which are not hyperbolic [21].

\section{Characterization of generalized augmented links}

We will be analyzing twisting and twist regions in a knot diagram. Twist regions and generalized twist regions are defined carefully in [24]. We review definitions here for convenience.

Definition 2.1. Let $K$ be a link in $S^{3}$, and let $D$ be a diagram of the link. We may view $D$ as a 4 -valent graph with over-under crossing information at each vertex. A twist region of the diagram $D$ is a sequence of bigon regions of $D$ arranged end to end, which is maximal in the sense that there are no other bigons on either end of the sequence. A single crossing adjacent to no bigons is also a twist region.

We will assume throughout that the diagram is alternating within a twist region, else replace it with a diagram with fewer crossings in the twist region.

In a twist region of a diagram, two strands twist around each other maximally, as in figure 1(a), and bound a "ribbon" surface. 
(a)

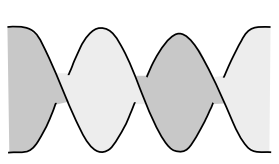

(b)

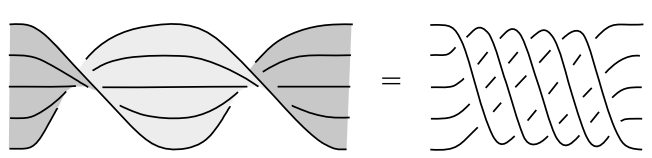

Figure 1: (a) A twist region. (b) A generalized twist region. Multiple strands lie on the twisted ribbon surface.

Definition 2.2. A generalized twist region of $D$ is a region of the diagram where two or more strands twist around each other maximally, as in figure 1(b). More precisely, a generalized twist region is a region of the diagram consisting of $m \geq 2$ parallel strands. When all the strands except the outermost two are removed from this region of the diagram, the remaining two strands form a twist region. In $S^{3}$, these two strands bound a ribbon surface between them. Remaining strands of the generalized twist region can be isotoped to lie parallel to each other, embedded on this ribbon surface.

The amount of twisting in each twist region is also important. We describe the amount of twisting in terms of half-twists and full-twists.

Definition 2.3. Let $K$ be a link in $S^{3}$. A half-twist of a generalized twist region of a diagram consists of a single crossing of the two outermost strands. The ribbon surface they bound, containing other strands of the twist region, flips over once in a half-twist.

A full-twist consists of two half-twists. Figure 1(b) shows a single fulltwist, or two half-twists, of five strands.

Given a diagram of a link in $S^{3}$, group crossings into generalized twist regions, such that each crossing is contained in exactly one generalized twist region. Call such a choice of generalized twist regions a maximal twist region selection. Note the choice is not necessarily unique. For example, in figure 1(b), we could group the crossings shown into a single generalized twist region containing a full-twist of five strands, or into 20 regular twist regions, each containing a single half-twist of two strands. Either choice is a valid maximal twist region selection, although the former seems more correct.

Now, at each generalized twist region in the maximal twist region selection, insert a crossing circle, that is, a simple closed curve $C_{i}$ encircling the strands of the twist region, and bounding a disk $D_{i}$ in $S^{3}$, perpendicular to the projection plane. The $D_{i}$ are called twisting disks. See figure 2(a). We can 
(a)

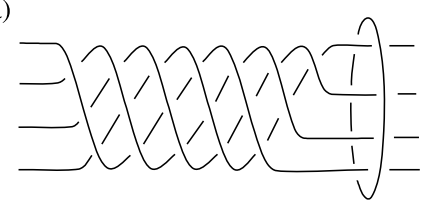

(b)

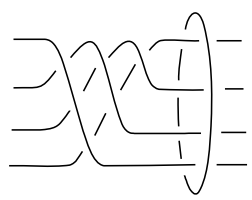

Figure 2: (a) Encircle each twist region with a crossing circle. (b) Link $L$ given by removing full-twists from the diagram.

select the $C_{i}$ and the $D_{i}$ such that the collection of all $D_{i}$ is a collection of disjoint disks in $S^{3}$.

When crossing circles are inserted at each twist region in the maximal twist region selection, we obtain a new link, with components $K_{j}$ from the original link $K$, and crossing circles $C_{i}$. The complement of this link is homeomorphic to the complement of the $\operatorname{link} L$ obtained by untwisting at each $C_{i}$. That is, we may remove all full-twists from each generalized twist region of the link diagram without changing the homeomorphism type of the link complement. See figure $2(\mathrm{~b})$.

The resulting diagram of $L$ consists of unknotted link components $C_{i}$ and components obtained from untwisting $K$, which we will call $K_{1}, \ldots, K_{p}$. In the diagram of $L$, the components of $K$ will either lie flat on the projection plane, or may have single half-twists encircled by crossing circles.

Definition 2.4. We call the link $L$ an augmentation of the diagram $D$ of $K$, or we say $L$ is the augmentation of the diagram $D$ corresponding to a maximal twist region selection. We also say that $L$ is obtained by augmenting $K$, and that $L$ is an generalized augmented link.

For brevity, we often drop the adjective "generalized" from the term generalized augmented links, since all augmented links we discuss here are of this form.

The connection between $S^{3}-L$ and the original link complement is given by Dehn filling. Any slope $s$ on a torus $T^{2}$ is parameterized by two relatively prime integers $p, q$, where $s=p \mu+q \lambda$, and $\mu, \lambda$ generate $H_{1}\left(T^{2} ; \mathbb{Z}\right)$. When $M$ is the link complement $S^{3}-L$, at the $i$ th crossing circle $C_{i}$, let $\mu_{i}, \lambda_{i}$ denote the meridian and longitude of $\partial N\left(C_{i}\right)$, respectively. Then Dehn filling along the slope $\mu_{i}+n_{i} \lambda_{i}$ gives a new link whose diagram no longer contains $C_{i}$, and the strands previously encircled by $C_{i}$ run through $n_{i}$ fulltwists (see, for example, Rolfsen [25]). Thus Dehn filling connects $S^{3}-K$ and the complement of the augmented link $L$. 


\subsection{Reflection}

The link $L$ admits a reflection, as follows. Arrange the diagram of $L$ such that crossing circles of $L$ lie perpendicular to the projection plane, and reflect the diagram of $L$ in the projection plane. The crossing circle components $C_{i}$ are taken to themselves. Outside of twist regions, the diagram of $L$ is preserved. If the components $K_{j}$ lie flat on the projection plane, they are also preserved by the reflection.

If some components $K_{j}$ run through a single half-twist at a twist region, then the reflection will reverse all the crossings of the half-twist, changing the direction of half-twist. Apply a twist homeomorphism, twisting one full twist at each half-twist in the opposite direction. This reverses the direction of the half-twist. Thus the composition of the reflection and the twist homeomorphism is an orientation reversing involution of $S^{3}-L$.

There is a surface that can be isotoped to be fixed pointwise by this involution, as follows. Outside of neigbhorhoods of half-twists, the surface consists of the projection plane. Inside a half-twist, the surface consists of the ribbon surfaces parallel to the knot strands, as well as another portion of surface. This other portion has boundary on the crossing circle, which we denote $C_{i}$, and on the outermost strands of the knots running through the half-twist. The boundary on $C_{i}$ is shown by the dotted line in figure 3 . It runs along the projection plane on the outside of $C_{i}$, so that the surface meets up with the projection plane outside a neighborhood of the halftwist. On the inside, this portion of the surface consists of two rectangles. One of these rectangles has a side attached to half of a longitude running up over the crossing circle. The opposite side is attached to the knot strand running up above the projection plane, crossing over the half-twist. The other two sides are joined to the surface of the projection plane outside the neighborhood of the half-twist, on the front left in figure 3 and on the back right. Similarly, there is another rectangle attached below the projection
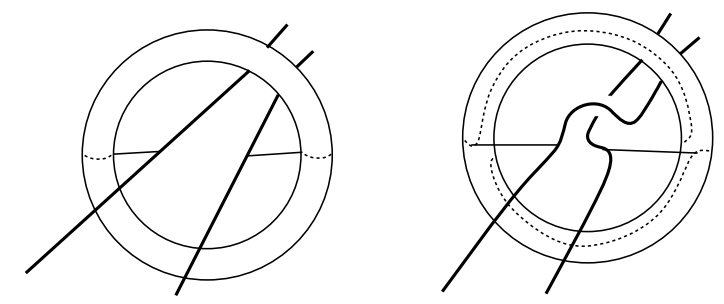

Figure 3: Left: $P \cap \partial N\left(C_{i}\right)$ has two components, shown in dotted lines. Right: $P \cap \partial N\left(C_{i}\right)$ has one component, giving a half-twist. 
plane, with two opposite sides running along the knot strand and along a half of a longitude, respectively, and the other two opposite sides attached to the surface of the projection plane on the front right in figure 3 and on the back left.

The above discussion illustrates the following, which is also Proposition 3.1 of [24].

Proposition 2.1. Let $L$ be an augmentation of a diagram of a link in $S^{3}$. Then $S^{3}-L$ admits a reflection, i.e. an orientation reversing involution for which the fixed point set is a surface.

\section{Slope lengths and hyperbolicity}

In this section, we prove results on slope lengths of generalized augmented links. Our methods generalize to hyperbolic manifolds which admit a reflection, and we state the more general results.

Lemma 3.1. Let $M$ be a 3-manifold with torus boundary components with the following properties:

(1) $M$ admits an orientation reversing involution $\sigma$ whose fixed point set is a properly embedded surface $P$ in $M$.

(2) Some boundary component $T$ of $M$ meets $P$, and for some slope $\lambda$ on $T, \sigma$ is an orientation reversing involution of $\lambda$. $($ Write $\sigma(\lambda)=-\lambda$.)

Then $\lambda$ meets $P$ exactly twice.

When our manifold is in fact a generalized augmented link, $\lambda$ may be the slope $\partial D_{i}$ on $\partial N\left(C_{i}\right)$, for example, or a slope $\partial D_{i}$ on $\partial N\left(K_{j}\right)$.

Proof. Since $\sigma$ takes $\lambda$ to $-\lambda$, a representative of $\lambda$ (which, by abuse of notation, we will also call $\lambda$ ) has a fixed point under $\sigma$. Thus $\lambda$ meets $P$. Additionally, since the only orientation reversing involutions of $S^{1}$ that fix a point must actually fix two points, $\lambda$ must meet $P$ twice.

Lemma 3.2. Let $M$ be as in Lemma 3.1. Then the torus $T$ is tiled by rectangles, each with one side parallel to the surface $P$, and one side orthogonal to $P$. The lift of these rectangles to the universal cover $\widetilde{T}$ gives a rectangular lattice in $\mathbb{R}^{2}$.

Proof. Consider the universal cover $\mathbb{R}^{2}$ of the torus boundary component $T$. As $P$ is embedded, the slopes $P \cap T$ lift to give parallel lines in $\mathbb{R}^{2}$. A simple 
curve representing the slope $\lambda$ lifts to give parallel lines perpendicular to the lines from $P$, since $\lambda$ is taken to $-\lambda$ by the involution $\sigma$ fixing $P$. The projection of these lines to $T$ gives a tiling of $T$ by rectangles. Together, the intersection points of these sets of lines form a lattice $\mathbb{Z}^{2}$ of $\mathbb{R}^{2}$.

Construct a basis of the lattice of Lemma 3.2 by letting $\boldsymbol{p}$ be a step parallel to a side from $P \cap T$, and by letting $\boldsymbol{o}$ be a step orthogonal to $\boldsymbol{p}$.

Lemma 3.3. Let $M$ be as in Lemma 3.1, and let $\{\boldsymbol{p}, \boldsymbol{o}\}$ be the basis for the lattice on $\widetilde{T}$ as above. Then the curve $\lambda$, which serves as one generator of $H_{1}(T ; \mathbb{Z})$, is given by $2 \boldsymbol{o}$. Another generator of $H_{1}(T ; \mathbb{Z})$ is given by $\boldsymbol{p}+\epsilon \boldsymbol{O}$, where $\epsilon=0$ if there are two components of $P \cap T$, and $\epsilon=1$ if there is one component of $P \cap T$.

Proof. By Lemma 3.1, $\lambda$ intersects $P$ twice. Thus its representative must cross lifts of $P$ twice in the lattice, and be taken to itself under the involution in $P$, so it is $2 \boldsymbol{o}$.

Note this implies that all corners of the rectangles formed by $\boldsymbol{p}$ and $\boldsymbol{o}$ project to just two distinct points on $T$ under the covering transformation. These two points are the projection of the endpoint of $\boldsymbol{o}$ and the projection of the endpoint of $2 \boldsymbol{o}$. Additionally, the fact that $\lambda=2 \boldsymbol{o}$ implies that $T$ is tiled by exactly two rectangles. To determine generators of $H_{1}(T ; \mathbb{Z})$, we determine whether these rectangles are glued with or without shearing on $T$.

Another obvious closed curve on $T$ besides $\lambda$ is given by a single component of $P \cap \partial T$. Call the corresponding slope $\alpha$. It does not necessarily generate $H_{1}(T ; \mathbb{Z})$ with $\lambda$. Since $\lambda$ intersects $P$ twice, either $\alpha$ intersects $\lambda$ once, in which case $P \cap T$ has two components, there is no shearing, and $\boldsymbol{p}$ is a generator; or $\alpha$ intersects $\lambda$ twice, and $P \cap T$ has one component.

If $P \cap T$ has one component, then $\alpha=2 \boldsymbol{p}$, and $\alpha$ is not a generator with $\lambda$. Then the endpoint of $\boldsymbol{p}$ must project to the same point as the endpoint of $\boldsymbol{o}$ under the covering projection, so $\boldsymbol{p}+\boldsymbol{o}$ will give a closed curve on $T$. Since it has intersection number 1 with $2 \boldsymbol{o}=\lambda, \boldsymbol{p}+\boldsymbol{o}$ will be a generator.

When $M$ is known to admit a hyperbolic structure, we can find lower bounds on the lengths of the $\operatorname{arcs} \boldsymbol{o}$ and $\boldsymbol{p}$ in the lattice. Recall that when a manifold has multiple cusps, lengths depend on a choice of maximal cusps, i.e., a collection of disjoint horoball neighborhoods, one for each cusp. Lengths of arcs are measured on the horospherical tori that form the boundaries of the horoball neighborhoods. To ensure lengths on a torus boundary are long, we need to ensure that we can choose maximal cusps appropriately. 
Theorem 3.1. Let $M$ be a 3-manifold with torus boundary components which admits a complete finite volume hyperbolic structure, and has the following additional properties:

(1) $M$ admits an orientation reversing involution $\sigma$ whose fixed point set is an embedded surface $P$ in $M$.

(2) Boundary components $T_{1}, \ldots, T_{t}$ of $M$ meet $P$, and for each $T_{i}$, there is a slope $\lambda_{i}$ that is taken to $-\lambda_{i}$ under $\sigma$.

Let $\left\{\boldsymbol{p}_{i}, \boldsymbol{o}_{i}\right\}$ generate the lattice on the universal cover $\widetilde{T}_{i}$ of $T_{i}$, of intersections of lines which project to $P$ and lines which project orthogonal to $P$, respectively, as in Lemma 3.3. Then there exists a choice of maximal cusps of $M$ such that, when measured on these maximal cusps, the length of each $\boldsymbol{o}_{i}$ is at least 1 , and the length of $\boldsymbol{p}_{i}$ is at least $1 / 2$.

Proof. Lift to the universal cover $\mathbb{H}^{3}$, which we view as the upper half space $\mathbb{H}^{3}=\{(x, y, z) \mid z>0\}$. For any $j$, we may apply an isometry of $\mathbb{H}^{3}$ such that the cusp corresponding to $T_{j}$ lifts to a horoball centered at the point at infinity.

By Mostow-Prasad rigidity, the involution of $M$ is isotopic to an isometry of $M$ under the hyperbolic metric. The surface $P$, since it is fixed pointwise, is isotopic to a totally geodesic surface in $M$ (see for example $[17,18]$ ). Thus it lifts to a collection of disjoint, totally geodesic planes.

Since $P$ meets the cusp corresponding to $T_{j}$, copies of $P$ will lift in $\mathbb{H}^{3}$ to parallel vertical planes through infinity. Because $P$ is fixed under the involution $\sigma$, the collection of parallel vertical planes must be preserved by a reflection of $\mathbb{H}^{3}$ in any one of the planes. Hence the (Euclidean) distance between any two adjacent planes is constant.

Now, define the horoball expansion about cusps of $T_{1}, \ldots, T_{t}$ as follows. For each $j$, select horoballs such that the lengths of the $\boldsymbol{o}_{j}$ agree for every $j$ simultaneously. That is, there exists some $\epsilon>0$ such that when each $\boldsymbol{o}_{j}$ has length $\epsilon$, the horoballs about the cusps corresponding to $T_{1}, \ldots, T_{t}$ are disjoint. Continue to increase $\epsilon$ keeping all the $\boldsymbol{o}_{j}$ of equal length, until it is as large as possible. We will show that the length of $\boldsymbol{o}_{j}$ is at least 1 when measured on this set of disjoint cusps.

Suppose not. Suppose the length of each $\boldsymbol{o}_{j}$ is less than 1 in this horoball expansion. Since this length is maximal, horoballs about cusps projecting to some $T_{i}$ and $T_{j}$ must abut. Apply an isometry to $\mathbb{H}^{3}$ such that the cusp corresponding to $T_{i}$ lifts to a horoball centered at infinity in $\mathbb{H}^{3}$, with height 1 in the upper half space model. It will be tangent to some horoball $H$ over 
a point $w$ on the boundary $\mathbb{C}=\{(x, y, 0)\}$ of $\mathbb{H}^{3}$, where $H$ projects to the cusp corresponding to $T_{j}$. Note $H$ is a ball of Euclidean diameter 1 .

Now consider lifts of the planes $P$. Some of these correspond to vertical planes parallel to each other, with the (Euclidean) distance between each plane equal to the length of $\boldsymbol{o}_{i}$.

We are assuming the length of $\boldsymbol{o}_{i}$ is strictly less than 1. Because the diameter of $H$ is $1, H$ must intersect a vertical plane $\widetilde{P}$ projecting to $P$. Because the reflection through the plane $\widetilde{P}$ projects to an isometry of $M$, the image of $H$ under this reflection must be a horoball in $\mathbb{H}^{3}$ disjoint from all other horoballs in the lift of the maximal cusps. Thus if $H$ lies over some point $w$ which is not on the plane $\widetilde{P}$, then the image of $H$ under the reflection through $\widetilde{P}$ will give a horoball distinct from $H$, which intersects $H$. This is impossible.

So $H$ is centered at a point $w \in \mathbb{C}$ which lies on a plane $\widetilde{P}$ which projects to $P$. In $\mathbb{H}^{3}$, there are vertical planes parallel to $\widetilde{P}$ on either side, each of distance from $w$ equal to the length of $\boldsymbol{o}_{i}$, which is strictly less than 1 .

On the other hand, $H$ projects to the cusp corresponding to $T_{j}$, which also meets $P$. Thus in $\mathbb{H}^{3}$ there will be a collection of hemispheres tangent at $w \in \mathbb{C}$ which project to $P$. These will be disjoint from (the interiors of) the vertical planes, since all these planes project to $P$ as well, and $P$ has no self intersections. The distance along $H$ from the top of $H$ to the nearest of these hemispheres will also be the length of $\boldsymbol{o}_{i}$, given our choice of horoball expansion.

Since the distance along $H$ from the top of $H$ to its equator is exactly 1, and by assumption the length of $\boldsymbol{o}_{i}$ is less than 1, this means that the radius of one of these hemispheres projecting to $P$ is greater than $1 / 2$. But then this hemisphere will intersect a vertical plane projecting to $P$ in its interior. This is a contradiction.

Now we have a horoball expansion such that all the $\boldsymbol{o}_{j}$ are of the same length, and this length is at least 1 , but this is not necessarily a choice of maximal cusps of $M$. It may be possible to expand some horoballs further. To finish the proof, starting with the given horoball expansion, choose any method of further expansion of horoballs such that when we have finished, each horoball neighborhood of a cusp abuts either itself or a horoball neighborhood of another cusp. This is a choice of maximal cusps. Since further expansion of any horoball only increases the length of arcs on the boundary of that horoball, the length of each $\boldsymbol{o}_{j}$ is still at least 1 .

To show the length of $\boldsymbol{p}_{j}$ is at least $1 / 2$, we consider the cusp corresponding to $T_{j}$. Because we have a choice of maximal cusps, when we lift to $\mathbb{H}^{3}$ such that this cusp lifts to a horoball about infinity, it must meet 
some full sized horoball $H$ corresponding to the lift of some (not necessarily different) cusp. Now, since $\boldsymbol{p}_{j}$ or $2 \boldsymbol{p}_{j}$ projects to a closed curve on $T_{j}$, a translation along $\boldsymbol{p}_{j}$ or $2 \boldsymbol{p}_{j}$ is a covering transformation. It must take $H$ to a disjoint horoball. Thus the translation length is at least 1 , so $\boldsymbol{p}_{j}$ has length at least $1 / 2$.

We wish to study what happens when we twist along the disks $D_{1}, \ldots, D_{t}$, i.e., when we perform Dehn filling on slopes $1 / n_{1}, \ldots, 1 / n_{t}$ on the cusps corresponding to $C_{1}, \ldots, C_{t}$, respectively. First, we give the following result about the lengths of such slopes. Note the following theorem applies to links in general 3-manifolds, not just $S^{3}$.

Proposition 3.1. Let $L=C_{1} \cup \ldots C_{t}$ be a link in a 3-manifold $M$, such that $M-L$ admits a complete, finite volume hyperbolic structure, admits an orientation reversing involution $\sigma$ whose fixed point set is a surface $P$, and for each component $C_{i}$ of $L$, there is a slope $\lambda_{i}$ taken to $-\lambda_{i}$ by $\sigma$.

Let $\mu_{i}$ be the other generator of $H_{1}\left(\partial N\left(C_{i}\right)\right)$ as in Lemma 3.3. Then the slope $\mu_{i}+n_{i} \lambda_{i}$ has length at least $\sqrt{(1 / 4)+c_{i}^{2}}$. Here:

(1) $c_{i}=2\left|n_{i}\right|$ if $P \cap \partial N\left(C_{i}\right)$ consists of two curves, or

(2) $c_{i}=2\left|n_{i}\right|-1$ if $P \cap \partial N\left(C_{i}\right)$ consists of one curve.

Proof. $M-L$ fits the requirements of the lemmas above. So in particular, by Lemma $3.3, H_{1}\left(\partial N\left(C_{i}\right) ; \mathbb{Z}\right)$ is generated by $2 \boldsymbol{o}_{i}$ and $\boldsymbol{p}_{i}+\epsilon_{i} \boldsymbol{o}_{i}$, where the generator $2 \boldsymbol{o}_{i}$ corresponds to the curve $\lambda_{i}$. If $P \cap \partial N\left(C_{i}\right)$ has two components, then one such component is a generator $\boldsymbol{p}_{i}=\mu_{i}$, and if $P \cap \partial N\left(C_{i}\right)$ has one component, then the other generator is $\boldsymbol{p}_{i}+\boldsymbol{o}_{i}=\mu_{i}$.

Suppose first that $P \cap \partial N\left(C_{i}\right)$ has two components. Then the slope $\mu_{i}+n_{i} \lambda_{i}$ is given by $\boldsymbol{p}_{i}+n_{i}\left(2 \boldsymbol{o}_{i}\right)$. Since $\boldsymbol{p}_{i}$ and $\boldsymbol{o}_{i}$ are orthogonal, by Theorem 3.1 this slope has length at least $\sqrt{(1 / 4)+4 n_{i}^{2}}=\sqrt{(1 / 4)+c_{i}^{2}}$.

Now suppose that $P \cap \partial N\left(C_{i}\right)$ has one component. Then the slope $\mu_{i}+$ $n_{i} \lambda_{i}$ is given by $\boldsymbol{p}_{i}+\boldsymbol{o}_{i}+n_{i}\left(2 \boldsymbol{o}_{i}\right)=\boldsymbol{p}_{i}+\left(1+2 n_{i}\right) \boldsymbol{o}_{i}$. It must have length at least $\sqrt{(1 / 4)+\left(1-2\left|n_{i}\right|\right)^{2}}=\sqrt{(1 / 4)+c_{i}^{2}}$.

Definition 3.1. If $P \cap \partial N\left(C_{i}\right)$ consists of one curve, as in case (2) of Proposition 3.1, we say there is a half-twist at $D_{i}$.

This terminology comes from considering a neighborhood of $D_{i}$ in $M$. In this neighborhood, a half-twist at $D_{i}$ is identical to a neighborhood of a half-twist of an augmented link in $S^{3}$, as in Definition 2.3. See figure 3. 
Two half-twists in a row in a neighborhood of $D_{i}$ again yields a fulltwist in this neighborhood. Thus Proposition 3.1 implies that the squared length of the slope $\mu_{i}+n_{i} \lambda_{i}$ on $C_{i}$ is at least one more than the squared number of half-twists inserted at $D_{i}$.

Theorem 3.2. Let $K$ be a knot or link in $S^{3}$ which has a diagram $D$ and a maximal twist region selection with at least six half-twists in each generalized twist region, and such that the corresponding augmentation is hyperbolic. Then $S^{3}-K$ is also hyperbolic.

Proof. The augmentation is a link with hyperbolic complement, by assumption. It admits an orientation reversing involution $\sigma$ fixing a surface $P$, and the cusps corresponding to crossing circles each have a slope $\lambda_{i}$ which is taken to $-\lambda_{i}$ by $\sigma$ : namely, the slope of the longitude of the crossing circle.

The original knot or link complement is obtained from this link complement by Dehn filling slopes on crossing circles. The longitude of a crossing circle is given by $\lambda_{i}$. The meridian is the generator $\mu_{i}$ of Proposition 3.1. If the knot has $c_{i}$ half-twists in the $i$ th twist region, then the Dehn filling slope is $\mu_{i}+n_{i} \lambda_{i}$, where $n_{i}=c_{i} / 2$ if $c_{i}$ is even, $n_{i}=\left(c_{i}+1\right) / 2$ if $c_{i}$ is odd.

By Proposition 3.1, the slope of the Dehn filling has length at least $\sqrt{(1 / 4)+c_{i}^{2}}>6$, since the diagram of $K$ has at least six half-twists in each generalized twist region. Thus by the six-Theorem $[2,15]$, the manifold resulting from Dehn filling is hyperbolike. By Perelman's proof of Geometrization, the manifold is hyperbolic.

\section{Volumes}

The existence of a reflection gives information about the volumes of augmented links as well. Theorem 4.1, below, is an immediate generalization of a similar theorem in [9].

Lemma 4.1. Let $K$ be a knot or link in $S^{3}$ which has a diagram $D$ and a maximal twist region selection such that the corresponding augmentation yields a link $L$ in $S^{3}$ whose complement is hyperbolic. Then the volume satisfies

$$
\operatorname{vol}\left(S^{3}-L\right) \geq 2 v_{8}(\operatorname{tw}(D)-1),
$$

where $v_{8} \approx 3.66386$ is the volume of a regular hyperbolic octahedron, and $\mathrm{tw}(D)$ is the number of generalized twist regions of the maximal twist region selection of $D$. 
Proof. By assumption, $S^{3}-L$ admits a complete hyperbolic structure. By Proposition 2.1, it admits a reflective symmetry. Thus $S^{3}-L$ contains a surface $P$ fixed pointwise under the reflection.

Cut $S^{3}-L$ along this surface. This produces a (possibly disconnected) manifold $N$ with totally geodesic boundary. By a theorem of Miyamoto [20], the volume of $N$ is at least $-v_{8} \chi(N)$, where $\chi(N)$ denotes the Euler characteristic of $N$.

Now, in the case that $P$ is the projection plane (i.e., no half-twists), cutting along $P$ splits $S^{3}-L$ into two balls, with half arcs corresponding to crossing circles drilled out of the ball. This is a handlebody. Since there are tw $(D)$ crossing circles, the genus of the handlebody is $\operatorname{tw}(D)$. Thus we obtain the volume estimate:

$$
\operatorname{vol}\left(S^{3}-L\right) \geq 2 v_{8}(\operatorname{tw}(D)-1)
$$

When the diagram has half-twists, let $L^{\prime}$ denote the link obtained by removing all half-twists from the diagram of $L$. Topologically, $S^{3}-L^{\prime}$ is obtained from $S^{3}-L$ by cutting $S^{3}-L$ along the disks bounded by crossing circles, and regluing with a half-twist.

Note $S^{3}-L^{\prime}$ has the following description as a gluing of ideal polyhedra. Cut $S^{3}-L^{\prime}$ along the projection plane. This slices each of the disks bounded by crossing circles in half. Now cut along each of these half disks and pull the disks apart. See figure 4.

This separates $S^{3}-L^{\prime}$ into two identical ideal polyhedra with faces given by crossing disks and by the projection plane. We may glue these polyhedra back in the manner in which we cut them to obtain $S^{3}-L^{\prime}$. We may also change the gluing on crossing disks only to obtain $S^{3}-L$, as follows. Rather than glue crossing disks straight across where $L$ has a half-twist, glue a half crossing disk on one polyhedron to the opposite half crossing disk on the opposite polyhedron, inserting the half-twist. See figure 5.
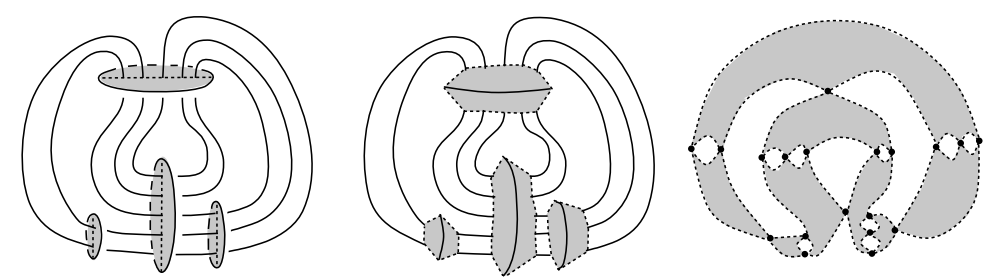

Figure 4: Decomposing $S^{3}-L^{\prime}$ into ideal polyhedra. First, cut along $P$. Second, cut along half disks. Finally, shrink remaining link components to ideal vertices. 

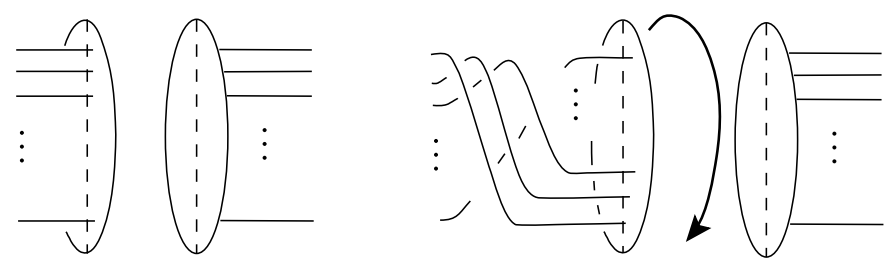

Figure 5: Left: Gluing without a half twist. Right: Inserting a half-twist.

Compute the Euler characteristic of the cut manifold $\left(S^{3}-L\right)-P$ by reading it off this polyhedral decomposition. Since $\left(S^{3}-L\right)-P$ has boundary, it retracts onto a one-skeleton. Build the one-skeleton by including a vertex for each ideal polyhedron (two vertices). Edges run through the half crossing disks which we glue. There will be one edge per glued pair of half crossing disks. Since there are tw $(D)$ crossing disks, the Euler characteristic is $2-2 \mathrm{tw}(D)$. Thus by Miyamoto's theorem, the volume satisfies: $\operatorname{vol}\left(S^{3}-L\right) \geq 2 v_{8}(\operatorname{tw}(D)-1)$.

Lemma 4.1 should be compared to Proposition 3.1 of [9]. The proof above is an immediate extension of the proof of that theorem to this more general case. For links with two strands per twist region, we showed in [9] that Lemma 4.1 is sharp.

In general, when crossing circles have more than two strands per twist region, Lemma 4.1 seems to actually be far from sharp. With Futer and Kalfagianni we have been able to develop better bounds on volumes of a certain class of knots [8]. Meanwhile, Lemma 4.1 gives a working lower bound on volumes.

Theorem 4.1. Let $K$ be a knot or link in $S^{3}$ which has a diagram $D$ and a maximal twist region selection with at least seven half-twists in each generalized twist region, and such that the corresponding augmentation is hyperbolic. Let $\operatorname{tw}(D)$ denote the number of generalized twist regions in the maximal twist region selection. Then

$$
\operatorname{vol}\left(S^{3}-K\right) \geq 0.64756(\operatorname{tw}(D)-1)
$$

Proof. Let $L$ be the augmentation, $S^{3}-L$ hyperbolic, by assumption. By Lemma 4.1, the volume satisfies:

$$
\operatorname{vol}\left(S^{3}-L\right) \geq 2 v_{8}(\operatorname{tw}(D)-1)
$$


Now, $S^{3}-K$ is obtained by Dehn filling $S^{3}-L$. Since there are at least seven half-twists per twist region, by Proposition 3.1, the Dehn filling is along slopes of length at least $\sqrt{49.25}>2 \pi$. Apply Theorem 1.1 of [9]. This theorem states that if $M$ is a hyperbolic manifold, and $s_{1}, \ldots, s_{k}$ are slopes on cusps of $M$ with minimum length $\ell_{\min }$ at least $2 \pi$, then the Dehn filled manifold $M\left(s_{1}, \ldots, s_{k}\right)$ is hyperbolic with volume bounded below by

$$
\operatorname{vol}\left(M\left(s_{1}, \ldots, s_{k}\right)\right) \geq\left(1-\left(\frac{2 \pi}{\ell_{\min }}\right)^{2}\right)^{3 / 2} \operatorname{vol}(M)
$$

In our case, $\ell_{\min } \geq \sqrt{49.25}$ and the volume of the unfilled manifold $S^{3}-L$ satisfies $\operatorname{vol}\left(S^{3}-L\right) \geq 2 v_{8}(\operatorname{tw}(D)-1)$. Thus the volume of $S^{3}-K$ satisfies

$$
\begin{aligned}
\operatorname{vol}\left(S^{3}-K\right) \geq & \left(1-\left(\frac{2 \pi}{\sqrt{49.25}}\right)^{2}\right)^{3 / 2} 2 v_{8}(\operatorname{tw}(D)-1) \\
& >0.64756(\operatorname{tw}(D)-1)
\end{aligned}
$$

\section{Geodesics}

We now give information on classes of geodesics in knot complements. Our tools are those of cone manifolds and cone deformations. We briefly review the definitions and results we use.

Definition 5.1. A hyperbolic cone manifold is a 3-manifold $M$ and a link $\Sigma$ in $M$ such that $M-\Sigma$ admits an incomplete hyperbolic metric, with cone singularities along $\Sigma$. That is, a neighborhood of $\Sigma$ in $M$ has a metric whose cross section is a hyperbolic cone, with cone angle $\alpha$ at the core.

A hyperbolic cone deformation is a one-parameter family of hyperbolic cone manifold structures on $M-\Sigma$.

In special cases, a Dehn filling can be realized geometrically as a cone deformation, as follows. Suppose $M$ is a 3-manifold with torus boundary which admits a complete hyperbolic metric. Let $s$ be a slope on $\partial M$. Then we may view the complete hyperbolic structure on $M$ as a hyperbolic cone manifold structure on $M(s)$ with cone angle zero along the link at the core of the attached solid torus in $M(s)$. 
We may always increase the cone angle from $\alpha=0$ to $\alpha=\varepsilon$, for some $\varepsilon>0$ via cone deformation, by work of Hodgson and Kerckhoff [11]. When $\alpha=\varepsilon$, in the hyperbolic cone metric, the slope $s$ will bound a singular disk. That is, a representative of $s$ can be isotoped to bound a disk $D$ which admits a smooth hyperbolic metric everywhere except at the core of $D$, where $D$ intersects the singular locus $\Sigma$. Thus this manifold with the hyperbolic cone metric is homeomorphic to $M(s)$.

In case there is a cone deformation starting at cone angle $\alpha=0$ and extending to $\alpha=2 \pi$, the final structure when $\alpha=2 \pi$ gives a complete, nonsingular hyperbolic metric on the manifold $M(s)$. In this case, we say the Dehn filling is realized by cone deformation.

The benefit of a cone deformation is that one obtains some geometric control on the hyperbolic structure of the manifold. In particular, when we have a single filling slope, the core of the Dehn filled solid torus is a closed geodesic in the hyperbolic structure given by cone angle $\alpha=2 \pi$. Thus this core is isotopic to a geodesic provided we can show a Dehn filling is realized by cone deformation.

Hodgson and Kerckhoff analyzed conditions which guarantee the existence of a cone deformation [12]. We will apply their results, but first we need the following definition.

Definition 5.2. Let $M$ be a 3-manifold with torus boundary $\partial M=T$ admitting a complete hyperbolic metric. Let $s$ be a slope on $T$. In the hyperbolic structure on $M, T$ becomes a cusp. Take any embedded horoball neighborhood of this cusp and consider its boundary. This inherits a Euclidean metric from the hyperbolic structure on $M$. Thus we may measure the length of $s$ and the area of the Euclidean torus $T$ with respect to this metric.

Define the normalized length of $s$ to be

$$
\ell_{\text {norm }}(s)=\frac{\operatorname{length}(s)}{\sqrt{\operatorname{area}(\mathrm{T})}} .
$$

Here length(s) is the length of a geodesic representing $s$. Note that unlike the lengths of Theorem 3.1, the normalized length of a slope is independent of choice of horoball neighborhood about the cusp corresponding to $T$.

The following is a consequence of Theorem 1.2 of [13].

Theorem 5.1 (Hodgson-Kerckhoff). Consider a complete, finite volume hyperbolic structure on the interior of a compact, orientable 3-manifold $M$ with $k \geq 1$ torus boundary components. Let $T_{1}, \ldots, T_{k}$ be horospherical 
tori which are embedded as cross-sections to the cusps of the complete structure. Let $s_{1}, \ldots, s_{k}$ be slopes, $s_{i}$ on $T_{i}$. Then $M\left(s_{1}, \ldots, s_{k}\right)$ admits a complete hyperbolic structure in which the core cures of the Dehn filled solid tori are isotopic to geodesics, provided the normalized lengths $\hat{L}_{i}=\ell_{\mathrm{norm}}\left(s_{i}\right)$ satisfy

$$
\sum_{i=1}^{k} \frac{1}{{\hat{L_{i}}}^{2}}<\frac{1}{(7.5832)^{2}}
$$

Theorem 1.2 of [13] is actually a more general theorem about Dehn filling space for manifolds with multiple cusps. However, in the proof of that theorem it is shown that under the above assumptions on normalized lengths of slopes, a cone deformation exists from cone angle 0 to $2 \pi$ for which each component of the singular locus has a tube of radius at least $\operatorname{arctanh}(1 / \sqrt{3})$ about it $[13$, p. 36]. The components of the singular locus correspond to the cores of the filled solid tori. Since each has a tube about it throughout the deformation, the cores remain isotopic to geodesics. See also the explanation in $[13,5]$, after the statement of Theorem 1.2 .

Lemma 5.1. Let $M, L, \lambda_{i}$ and $\mu_{i}$ be as in Proposition 3.1. Then the normalized length of each slope $s_{i}=\mu_{i}+n_{i} \lambda_{i}$ is at least

$$
\ell_{\text {norm }}\left(s_{i}\right) \geq \sqrt{c_{i}}
$$

where again $c_{i}$ is the number of half-twists inserted by the Dehn filling along slope $s_{i}$.

The proof of Lemma 5.1 is similar to that of Proposition 3.1, except with the added difficulty that we are considering normalized lengths, and not actual lengths. Compare [23, Proposition 6.5].

Proof. Write the slope $s_{i}=\mu_{i}+n_{i} \lambda_{i}$ in terms of the lengths of $\boldsymbol{o}_{i}$ and $\boldsymbol{p}_{i}$, of Lemma 3.3. In particular, as in Proposition 3.1, the slope is given by $\boldsymbol{p}_{i}+c_{i} \boldsymbol{o}_{i}$, where $c_{i}$ is the number of half-twists inserted by the Dehn filling, and since $\boldsymbol{o}_{i}$ and $\boldsymbol{p}_{i}$ are orthogonal, its length is given by $\sqrt{p_{i}^{2}+c_{i}^{2} o_{i}^{2}}$, where $p_{i}$ and $o_{i}$ denote the lengths of geodesic representatives of $\boldsymbol{p}_{i}$ and $\boldsymbol{o}_{i}$. By Lemma 3.3, the area of the cusp torus is given by $2 o_{i} p_{i}$.

Thus the normalized length of $s_{i}=\mu_{i}+n_{i} \lambda_{i}$ is given by

$$
\ell_{\mathrm{norm}}\left(s_{i}\right)=\frac{\sqrt{p_{i}^{2}+c_{i}^{2} o_{i}^{2}}}{\sqrt{2 p_{i} o_{i}}}=\sqrt{\frac{p_{i}}{2 o_{i}}+\frac{c_{i}^{2} o_{i}}{2 p_{i}}} .
$$


Minimize the normalized length with respect to $p_{i} / o_{i}$. We find that its value is minimum when the ratio $p_{i} / o_{i}$ equals $c_{i}$. In this case, the normalized length will be $\sqrt{c_{i}}$.

We may now prove Theorem 5.2, giving results on isotopy classes of geodesics in generalized augmented links.

Theorem 5.2. Let $K$ be a knot or link in $S^{3}$ which has a diagram $D$ and a maximal twist region selection with $\mathrm{tw}(D)$ twist regions, such that the corresponding augmentation is hyperbolic. Let $c_{i}$ be the number of half-twists in the $i$-th twist region. Then each crossing circle is isotopic to a geodesic in the hyperbolic structure on $S^{3}-K$, provided

$$
\sum_{i=1}^{\operatorname{tw}(D)} \frac{1}{c_{i}}<\frac{1}{(7.5832)^{2}}
$$

Proof. $S^{3}-K$ is obtained from $S^{3}-L$ by Dehn filling the crossing circles. By Lemma 5.1, the normalized lengths of the slopes of the Dehn filling are at least $\sqrt{c_{i}}$, where $c_{i}$ is the number of half-twists in the $i$ th generalized twist region of $D$. By Theorem 5.1, the cores of the filled solid tori are isotopic to geodesics provided

$$
\sum_{i=1}^{\operatorname{tw}(D)} \frac{1}{c_{i}}<\frac{1}{(7.5832)^{2}}
$$

\section{Acknowledgments}

Research was partially funded by NSF grant DMS-0704359. We thank David Futer and John Luecke for helpful conversations. We also thank the referee for many helpful comments, particularly for helping to streamline the proof of Theorem 3.1.

\section{References}

[1] C.C. Adams, Augmented alternating link complements are hyperbolic, 'Low-dimensional Topology and Kleinian Groups' (Coventry/Durham, 1984), London Math. Soc. Lecture Note Ser., 112, Cambridge University Press, Cambridge, 1986, 115-130.

[2] I. Agol, Bounds on exceptional Dehn filling, Geom. Topol. 4 (2000), 431-449 (electronic). 
[3] K.L. Baker, Surgery descriptions and volumes of Berge knots. I. Large volume Berge knots, J. Knot Theory Ramifications 17(1) (2008), 1077-1097.

[4] K.L. Baker, Surgery descriptions and volumes of Berge knots. II. Descriptions on the minimally twisted five chain link, J. Knot Theory Ramifications 17(9) (2008), 1099-1120.

[5] J. Berge, Some knots with surgery yielding lens spaces, unpublished manuscript.

[6] J. Birman and I. Kofman, A new twist on Lorenz links, arXiv:0707.4331.

[7] A. Champanerkar, I. Kofman and E. Patterson, The next simplest hyperbolic knots, J. Knot Theory Ramifications 13(1) (2004), 965-987.

[8] D. Futer, E. Kalfagianni and J.S. Purcell, On diagrammatic bounds of knot volumes and spectral invariants, arXiv:math/0901.0119.

[9] D. Futer, E. Kalfagianni and J.S. Purcell, Dehn filling, volume, and the Jones polynomial, J. Differential Geom. 78(3) (2008), 429-464.

[10] C.McA. Gordon and J. Luecke, Knots are determined by their complements, J. Amer. Math. Soc. 2(2) (1989), 371-415.

[11] C.D. Hodgson and S.P. Kerckhoff, Rigidity of hyperbolic cone-manifolds and hyperbolic Dehn surgery, J. Differential Geom. 48(1) (1998), 1-59.

[12] C.D. Hodgson and S.P. Kerckhoff, Universal bounds for hyperbolic Dehn surgery, Ann. Math. (2) 162(1) (2005), 367-421.

[13] C.D. Hodgson and S.P. Kerckhoff, The shape of hyperbolic Dehn surgery space, Geom. Topol. 12(2) (2008), 1033-1090.

[14] S.M. Kuhlmann, Geodesic knots in cusped hyperbolic 3-manifolds, Algebr. Geom. Topol. 6 (2006), 2151-2162 (electronic).

[15] M. Lackenby, Word hyperbolic Dehn surgery, Invent. Math. 140(2) (2000), 243-282.

[16] M. Lackenby, The volume of hyperbolic alternating link complements, Proc. London Math. Soc. (3) 88(1) (2004), 204-224, With an appendix by Ian Agol and Dylan Thurston.

[17] C.J. Leininger, Small curvature surfaces in hyperbolic 3-manifolds, J. Knot Theory Ramifications 15(3) (2006), 379-411. 
[18] W. Menasco and A.W. Reid, Totally geodesic surfaces in hyperbolic link complements, Topology '90 (Columbus, OH, 1990), Ohio State Univ. Math. Res. Inst. Publ., 1, de Gruyter, Berlin, 1992, pp. 215-226.

[19] S.M. Miller, Geodesic knots in the figure-eight knot complement, Experiment. Math. 10(3) (2001), 419-436.

[20] Y. Miyamoto, Volumes of hyperbolic manifolds with geodesic boundary, Topology 33(4) (1994), 613-629.

[21] J.S. Purcell, On multiply twisted knots that are Seifert fibered or toroidal, arXiv:0906.4575.

[22] J.S. Purcell, Volumes of highly twisted knots and links, Algebr. Geom. Topol. 7(2007), 93-108.

[23] J.S. Purcell, Cusp shapes under cone deformation, J. Differential Geom. 80(3) (2008), 453-500.

[24] J.S. Purcell, Slope lengths and generalized augmented links, Comm. Anal. Geom. 16(4) (2008), 883-905.

[25] D. Rolfsen, Knots and links, Publish or Perish Inc., Berkeley, Calif., 1976, Mathematics Lecture Series, No. 7.

Department of Mathematics

Brigham Young University

Provo

UT 84602

USA

E-mail address: jpurcell@math.byu.edu

RECEIVED July 14, 2009 